\title{
Téoros
}

Revue de recherche en tourisme

\section{Le rôle des centres locaux de développement en agrotourisme au Québec}

\section{Martin Cardinal}

Volume 20, numéro 2, été 2001

Tourisme rural

URI : https://id.erudit.org/iderudit/1071642ar

DOI : https://doi.org/10.7202/1071642ar

Aller au sommaire du numéro

Éditeur(s)

Université du Québec à Montréal

ISSN

0712-8657 (imprimé)

1923-2705 (numérique)

Découvrir la revue

Citer cet article

Cardinal, M. (2001). Le rôle des centres locaux de développement en

agrotourisme au Québec. Téoros, 20(2), 39-43. https://doi.org/10.7202/1071642ar d'utilisation que vous pouvez consulter en ligne.

https://apropos.erudit.org/fr/usagers/politique-dutilisation/ 


\section{Le rôle des centres locaux de développement en agrotourisme au Québec}

\section{Martin Cardinal}

\section{C} réés en 1997, les Centres locaux de développement (CLD), organismes à but non lucratif financés conjointement par le gouvernement et les municipalités, sont de nouveaux intervenants du monde touristique. Mentionnons que plusieurs CLD ont développé un volet de développement et de promotion touristiques (Association des Centres locaux de développement du Québec, 2001).

Les CLD peuvent intervenir non seulement en milieu urbain, mais également en milieu rural. Le territoire d'implantation d'un CLD est la Municipalité régionale de comté (MRC) ou son équivalent quand il n'y a pas de MRC. La mission d'un CLD est de mobiliser l'ensemble des acteurs locaux dans une démarche commune tournée vers l'action et son objectif est de favoriser le développement de l'économie et la création d'emplois, dans le cadre d'un partenariat entre le gouvernement et la communauté locale. Dans le but d'assurer l'efficacité et la cohérence du soutien à l'entrepreneuriat local, le gouvernement ne finance dorénavant, au palier local, que cet organisme de soutien aux entreprises. Le CLD prend donc une importance particulière pour l'entrepreneuriat du monde touristique.

De façon plus précise, les responsabilités d'un CLD consistent à :

- Regrouper ou coordonner les différents services d'aide à l'entrepreneuriat et à l'entreprise en concertant les organismes qui travaillent déjà dans ce domaine et assurer le financement de ces services.

- Élaborer un plan d'action local pour l'économie et l'emploi (PALÉE) et veiller à sa réalisation. Le CLD joue donc un rôle majeur dans l'animation du milieu local en matière de développement économique et de développement de l'emploi par la concertation de tous les intervenants concernés sur son territoire. Il est alors crucial pour l'industrie touristique de faire inscrire ses priorités sectorielles de développement dans cette stratégie, puisqu'il s'agit d'un plan triennal et que les ressources du CLD seront affectées en fonction de la concrétisation de ce plan.

- Élaborer, en tenant compte des stratégies nationales et régionales, toute stratégie locale liée au développement de l'entrepreneuriat et des entreprises, y compris les entreprises de l'économie sociale.

- Agir en tant que comité consultatif auprès du Centre local d'emploi (CLE) de son territoire et exécuter, sur autorisation du ministre, tout autre mandat provenant des ministères et des organismes gouvernementaux concernés par le développement local.

Ses champs d'intervention sont :

- la prospection de l'investissement et l'accueil,

- le soutien à l'entreprise locale,

- le soutien aux jeunes entrepreneurs,

- les dossiers spéciaux régionaux à caractère économique,

- la promotion du territoire et l'animation économique.

\section{Le rôle des agents ruraux de développement}

À la suite du Sommet sur l'économie et l'emploi, organisé par le Gouvernement du Québec en octobre 1996, le ministère des Régions, dont le mandat est d'assurer la coordination des interventions publiques en matière de développement des communautés rurales dans la province, a mis en place un programme intitulé Mesures de soutien au développement rural, dont un volet concerne l'engagement d'agents ruraux, en collaboration avec Solidarité rurale du Québec. Il s'agit d'une aide financière maximale de $20000 \$$ accordée sous la forme d'une subvention annuelle aux municipalités qui comptent au moins 5000 habitants et aux organismes de développement sans but lucratif. L'organisation bénéficiaire doit contribuer pour un montant équivalent et cette mesure est renouvelable si les conditions du protocole d'entente ont été respectées (Ministère des Régions, 2001).

Les agents ruraux de développement ont comme fonctions spécifiques :

- d'animer et de soutenir le développement sous toutes ses formes ;

- d'améliorer la connaissance que les milieux possèdent des ressources humaines et physiques de leur territoire ;

- de faire la promotion du développement intégré des ressources ;

- de favoriser l'émergence de projets et d'initiatives de développement dans les secteurs social, culturel, touristique, patrimonial, économique et communautaire ;

- de soutenir techniquement les promo- 


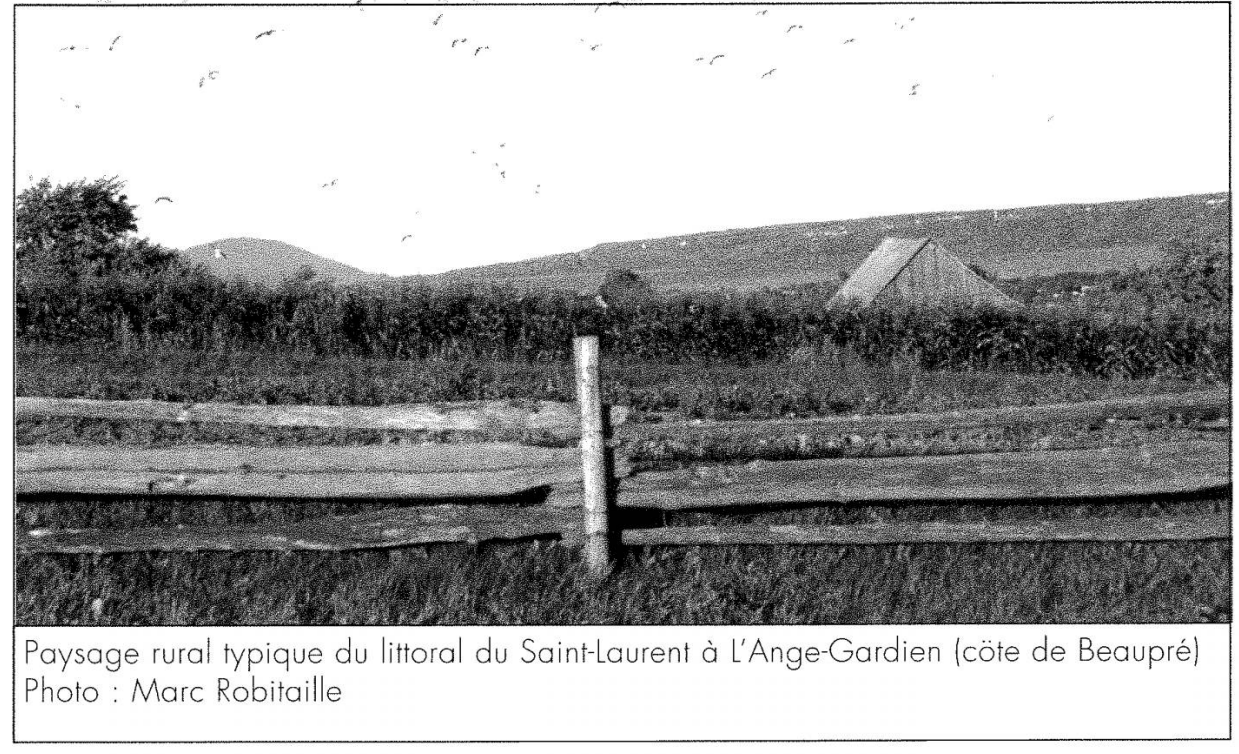

teurs individuels et collectifs dans l'élaboration de leurs projets.

Plusieurs agents ruraux de développement travaillent dans les CLD du Québec. Ces organismes définissent leur tâche en fonction du PALÉE. Il ne s'agit donc pas d'une mesure uniforme à la grandeur de la province, mais d'un programme que chaque communauté rurale adapte à ses priorités stratégiques de développement. Plusieurs d'entre elles donnent un mandat agrotouristique et de tourisme rural à 1'agent de développement rural, quand c'est un axe prioritaire du milieu.

Par exemple, au CLD Côte-de-Beaupré, l'agriculture, la foresterie et le tourisme font partie du mandat de l'agent de développement rural. En ce qui concerne le tourisme, l'agent veille au développement et à la promotion touristiques de la MRC. Essentiellement, sa tâche consiste à concerter le milieu touristique local afin de dégager des consensus sur certains dossiers - la réédition de la carte touristique, la création d'un portail Internet, la relocalisation du bureau d'information touristique et la confection d'un guide touristique spécifique, notamment - afin de les faire avancer.

Nous verrons, dans les paragraphes suivants, que l'intervention de l'agent de développement rural en agrotourisme doit tenir compte de plusieurs éléments dont l'offre et la demande agrotouristiques québécoises, la commercialisation de l'agrotourisme, la fidélisation des agrotouristes ainsi que les défis et les enjeux du développement de l'agrotourisme au Québec.

\section{L'offre agrotouristique québécoise}

S'il veut intervenir efficacement dans le milieu, l'agent de développement rural doit identifier correctement l'offre agrotouristique de sa région. Dans son action, il utilise une classification de l'offre agrotouristique en quatre catégories.

- La première catégorie, l'événement à caractère agricole, regroupe tous les festivals et les activités qui ont une thématique liée à l'agriculture (Le Groupe Type, 1997). La présence de touristes et la mise en valeur d'une production locale font en sorte de stimuler l'économie d'une région et de faire connaître ses particularités agricoles. Comme exemples de ce type d'événement nous pouvons, entre autres, citer la Fête des vendanges de Magog-Orford et le Festival de l'Érable de Plessisville.

- La deuxième catégorie, l'érablière, offre des repas champêtres dans un cadre naturel. Faisant partie de la tradition et du folklore québécois, les cabanes à sucre artisanales ou commerciales offrent parfois, en plus de la nourriture, une interprétation de l'acériculture.

- La troisième catégorie regroupe les cultures et la transformation agroalimentaire. On y trouve principalement les vignobles, les cidreries, les serres, les pépinières, les jardins, les vergers et les bleuetières qui permettent la cueillette et l'achat, ainsi que l'interprétation de leurs installations. Un exemple de ce type d'entreprise est le Musée de 1'Abeille-Économusée du miel de Château-Richer sur la Côte-de-Beaupré, où les gens peuvent faire une visite commentée sur le thème de l'abeille et du miel, voir le fonctionnement d'une ruche grâce à un safari-abeille et acheter des produits transformés sur place dans la boutique attenante au centre d'interprétation.

- La dernière catégorie, les fermes animalières et leurs services, regroupe trois produits distincts. Il y a d'abord les visites des élevages et des installations de la ferme principalement orientées vers les animaux exotiques. Le circuit des Grands gibiers domestiques de St-Patrice-de-Beaurivage dans la MRC de Lotbinière, en Chaudière-Appalaches, représente bien ce concept. Quatre fermes se sont associées (Fermes Baronets, Beaulieu, Clermont Labrecque et Fils ainsi que le Ranch Camden) pour créer un forfait de groupe original qui permet aux visiteurs d'aller dans les quatre exploitations et rencontrer directement les éleveurs d'animaux exotiques (autruche, bison, sanglier et cerf de Virginie). Il y a aussi les gîtes à la ferme, où les visiteurs participent aux travaux et entrent en contact direct avec la réalité agricole dans ses gestes quotidiens. Soulignons ici le programme Le Gîte à la Ferme de la Fédération des Agricotours du Québec qui consiste essentiellement en un service d'hébergement offert dans la maison d'un producteur agricole et qui comprend des activités liées à la ferme. Finalement, il y a la formule des repas champêtres (les touristes partagent un repas à la ferme) ou des tables champêtres (dont celles d'un autre programme des Agricotours nommé La Table champêtre qui comporte un service de repas, offert dans la 
maison du producteur agricole, composé principalement des produits de sa ferme et de produits régionaux).

\section{La demande agrotouristique québécoise}

Toujours afin de pouvoir intervenir dans le milieu rural, l'agent de développement rural doit également identifier la demande agrotouristique et le comportement des visiteurs qui pratiquent l'agrotourisme.

Le marché potentiel de l'agrotourisme au Québec peut être divisé en deux parties (Zins, 1997). D'abord, il y a le marché hors Québec (Zins, 1997) qui regroupe les touristes qui découvrent notre culture et notre géographie par le biais de l'agriculture et de la ruralité ; ce groupe inclut, entre autres, les agriculteurs étrangers. Puis, il y a le marché québécois, soit la clientèle primaire à développer.

Le potentiel agrotouristique québécois apparaît sous-exploité puisque 1'offre de produits est encore peu développée et peu diversifiée (Zins, 1997). Cette offre est d'ailleurs toujours au stade de l'émergence et deux éléments nuisent à la réalisation du plein potentiel de ce marché : le fait que «[...] le produit agrotouristique n'est pas encore mis en valeur de façon adéquate $[\ldots] »$ et qu'il « [...] reste encore mal connu, mal compris et mal positionné [...] »(Zins, 1997).

L'agent de développement rural doit aussi prendre en considération les principales caractéristiques de la clientèle agrotouristique. Cette clientèle provient majoritairement des milieux urbains (Montréal), est hautement scolarisée (études collégiales et universitaires), est relativement jeune (de 31 à 45 ans), a des revenus élevés, est mariée et a des enfants, a peu ou pas de contacts avec le milieu rural, bénéficie de plus d'une période de vacances par année et veut faire connaître la vie à la campagne à ses enfants (Fédération des Agricotours du Québec, 1984). De plus, les utilisateurs d'attraits agrotouristiques sont largement les excursionnistes et ils ne se considèrent pas nécessairement comme des agrotouristes (Girardville et coll., 1996). En ce qui con- cerne les motivations, on peut segmenter le marché en deux grands groupes de clients : les initiés à l'agriculture qui veulent acquérir des connaissances techniques dans le but ultime d'effectuer des transferts technologiques et les néophytes qui veulent simplement découvrir «un nouvel univers: l'espace rural et le producteur agricole » (Le Groupe Type, 1997).

Finalement, pour bien comprendre le comportement des visiteurs, on doit tenir compte du fait que la pratique de l'agrotourisme apparaît liée au désir de vivre le dépaysement, d'entrer en contact avec la nature, de découvrir le milieu de vie rural, d'entrer en relation avec le producteur agricole, de vivre une expérience authentique, de découvrir des produits régionaux originaux et artisanaux et d'admirer des paysages et des environnements de qualité (Le Groupe Type, 1997).

\section{La commercialisation de I'agrotourisme}

En tenant compte de l'offre et de la demande agrotouristiques, il est maintenant possible de définir quelques pistes d'interventions pour l'agent de développement rural.

Pour conserver le cachet spécial de l'agrotourisme, il faut absolument éviter la standardisation du produit. L'offre devrait plutôt refléter la production régionale et miser sur cette spécificité afin de demeurer la plus artisanale possible (Le Groupe Type, 1997). Le développement de produits régionaux distincts devrait se faire en harmonie avec le potentiel agroalimentaire local afin de bien le refléter, d'éviter le copiage de ce que les autres font et de saper les efforts de tout le monde. Enfin, pour conserver l'authenticité recherchée par les visiteurs, il faudrait le plus possible promouvoir le lien agriculteurtouriste et songer à créer une restauration axée sur les produits du terroir.

Si tous ces éléments sont correctement organisés, il y a de fortes chances que l'on réponde aux attentes des utilisateurs d'attraits agrotouristiques de manière appro- priée et que le marché se développe correctement et connaisse une croissance soutenue.

La mise en marché de l'agrotourisme au Québec sera une réussite dans la mesure où les outils de commercialisation seront utilisés à bon escient. On peut suggérer l'utilisation de différents outils ou moyens de commercialisation pour rejoindre les agrotouristes :

- Associations : En devenant membre de l'association touristique régionale, de l'office de tourisme, de la chambre de commerce, du centre local de développement ou de tout autre regroupement sectoriel pertinent, une entreprise agrotouristique profite de différents services professionnels et surtout d'une visibilité intéressante à peu de frais (apparition dans différents guides et répertoires, possibilité de participer à différentes opérations concertées de promotion) tout en adhérant à un réseau qui pourrait éventuellement lui procurer des occasions d'affaires intéressantes.

- Circuit régional ou thématique : Le fait de s'intégrer à un circuit composé de différents attraits touristiques distinctifs et complémentaires diminue les coûts publicitaires per capita et augmente l'attractivité d'une entreprise agrotouristique en développant une destination globale intéressante. Un exemple éloquent de ce type de produit touristique est Le Circuit du paysan, conçu par un agent de développement rural du Centre local de développement Haut-StLaurent et Jardins-de-Napierville, qui regroupe centres d'interprétation, vignobles, cidreries, vergers, fermes, etc.

- Guide touristique de l'association touristique régionale : Cet outil d'information est extrêmement utilisé par les touristes et il constitue la principale référence sur les différentes régions du Québec. Idéalement, quand cela est possible, il est avantageux d'y figurer spécifiquement dans la section thématique portant sur l'agrotourisme.

- Guide agrotouristique régional : $\mathrm{Ce}$ document est un « outil d'information unique pour connaître les ressources 


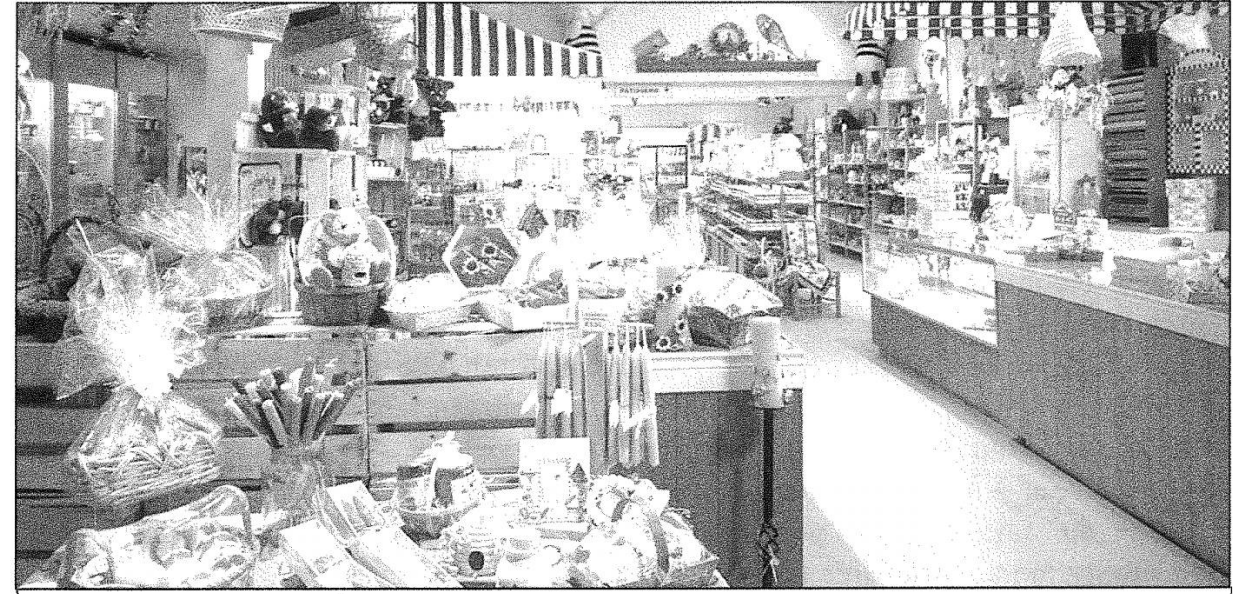

Le Musée de l'Abeille-Économusée du miel, situé à Château-Richer sur la côte de Beaupré, offre aux visiteurs, en plus du centre d'interprétation, une boutique de produits à base de miel.

Photo: Musée de l'Abeille-Économusée du miel

existantes » en matière d'agrotourisme (Le Groupe Type, 1997 : 5-31) dans une région donnée. S'il n'existe pas déjà dans leur région, les entreprises agrotouristiques devraient sérieusement songer à en créer un pour pallier le manque d'organisation de l'information dans ce secteur d'activité. Le SaguenayLac-Saint-Jean s'est doté d'un tel guide agrotouristique intitulé Le terroir des Bleuets depuis au moins 1999. Celui-ci se présente maintenant sous forme d'un dépliant dans lequel on trouve une carte positionnant l'offre agrotouristique régionale et différentes sections thématiques (hébergement à la ferme, table à la campagne, au pays des bleuets, à dos de cheval, à la découverte de la ferme, la cueillette de vos fruits et légumes, des animaux d'ici et d'ailleurs, les délices de chez nous, paradis de chasse et pêche, les petits kiosques à la ferme).

- Dépliant : Il est nécessaire qu'une entreprise touristique possède un dépliant pour se vendre auprès de ses visiteurs potentiels. Ce dépliant devrait être très invitant et représenter correctement les activités de l'entreprise. Le secret de son succès réside, entre autres, dans sa distribution stratégique. Celleci devrait au moins se faire dans les bureaux d'information touristique de la MRC, de la région touristique, des régions touristiques limitrophes, dans les centres infotouristes de Montréal et de Québec ainsi que dans les principaux établissements hôteliers et les gîtes touristiques des villes avoisinantes.

- Événements spéciaux : L'organisation d'événements spéciaux intégrés dans une programmation originale attire toujours l'attention du public. Les gens recherchent des choses nouvelles et différentes à faire. Pourquoi ne pas animer un attrait pour le rendre irrésistible? Certains sites s'y prêtent plus que d'autres. Par exemple, un centre d'interprétation de l'agriculture pourrait très bien organiser une journée spéciale sur les organismes modifiés génétiquement (OGM) avec des spécialistes sur place pour informer le public sur cette problématique très médiatisée depuis quelque temps.

- Médias et journaux : Les médias électroniques (télévision, radio, Internet...) et les médias écrits (journaux, revues...) s'adressent généralement à la masse locale et régionale. Ils visent donc la clientèle des excursionnistes. Il peut être bon, selon l'attrait touristique, d'utiliser ce moyen pour rejoindre le public cible.

- Bouche-à-oreille : C'est la meilleure publicité possible parce qu'elle ne coûte pas cher et qu'elle est très crédible. Pour la maximiser, un attrait agrotouristique doit miser sur la qualité du produit, son originalité et l'accueil exceptionnel offert (Le Groupe Type, 1997).

- Foires agricoles : Il est bon pour une entreprise agrotouristique d'avoir un stand dans une exposition agricole pour faire connaître son produit dans un contexte qui s'y prête bien. On peut raisonnablement croire que les visiteurs de tels événements aiment l'agriculture et tout ce qui s'y rapporte et qu'ils peuvent provenir de la région immédiate, de régions limitrophes ou carrément de l'extérieur. Il s'agit de clients potentiels pour un site à vocation agrotouristique. Participer à une exposition agricole comme Expo Québec qui dure plusieurs jours en plein été et où la participation populaire est impressionnante constitue certainement une vitrine de choix pour faire connaittre une entreprise agrotouristique et ses produits.

- Signalisation touristique : Il s'agit de la base, mais nombreux sont ceux qui négligent cet aspect fondamental. Comme l'écrit Jacques Demers dans un ouvrage traitant de développement touristique, les panneaux de signalisation «[...] influencent le public et une affiche bien faite est invitante » (1987: 231). Le contraire est aussi vrai. Il faut donc porter une attention toute particulière à la signalisation d'un attrait touristique si l'on veut attirer du monde. Il s'agit d'un dossier qui peut s'avérer compliqué parce qu'il peut être assez technique, dépendant de la réglementation de la localité concernée. Il faut donc bien s'informer des règlements en vigueur tant dans la municipalité visée, à la MRC et au ministère des Transports avant de procéder à l'installation de panneaux. Il est aussi sage de confier la confection des panneaux de signalisation à un spécialiste en la matière ; nombreux sont les exemples d'affiches inefficaces parce que fabriquées par des amateurs.

- Sites internet : Nous savons qu'aujourd'hui la clientèle utilise énormément l'Internet dans sa quête d'information et cela est d'autant plus vrai en tourisme. Conséquemment, il est presque indispensable pour une entreprise agrotouris- 
tique de posséder son site Internet ou du moins de figurer sur le site d'un regroupement ou d'une association. Il est également pertinent d'avoir une adresse électronique que les clients potentiels peuvent utiliser pour poser des questions. Cela augmente grandement l'accessibilité de l'entreprise agrotou-ristique, à peu de frais.

\section{Fidéliser les agrotouristes}

Grâce à ses interventions, l'agent de développement rural doit également considérer que la commercialisation ne peut se faire sans la recherche d'une relation prolongée entre l'entreprise et le touriste.

Si la clientèle est satisfaite de son expérience, elle reviendra ou deviendra la meilleure ambassadrice possible de l'attrait (Duhaine et coll., 1996). Si, au contraire, elle est insatisfaite, la clientèle se tournera vers d'autres qui répondront à ses besoins et à ses attentes de façon concluante, surtout dans le domaine touristique. Comme les entreprises agrotouristiques comptent beaucoup sur le boucheà-oreille comme mode de communication peu dispendieux, il faut réduire au minimum les cas de personnes insatisfaites, car elles sont des détracteurs potentiels qui peuvent causer des torts immenses à l'entreprise.

Pour s'assurer que le client est réellement satisfait en bout de ligne, il faut s'efforcer de lui vendre l'attrait agrotouristique en suscitant chez lui des attentes réalistes face au produit proposé.

\section{Les défis et les enjeux du développement de I'agrotourisme au Québec}

Bien que l'agrotourisme semble être une opportunité de développement intéressante pour la plupart des régions du Québec, il convient d'en examiner lucidement les conséquences avant d'aller de l'avant avec un tel projet. Du côté des avantages, on peut mentionner que cela représente des revenus additionnels à la ferme, un réseau de distribution pour les productions agricoles, une façon de faire connaître le métier d'agriculteur, une manière d'échanger avec les citadins et de faire comprendre la nécessité de cette activité économique (Le Groupe Type, 1997). En ce qui concerne les inconvénients, mentionnons le danger pour l'environnement, la menace socioculturelle, les problèmes fonciers (schéma d'aménagement, zonage agricole), la formation nécessaire des agriculteurs (techniques d'accueil, marketing), le peu de profits pour ceux qui n'offrent que des visites et la concurrence avec les travaux agricoles en saison (Le Groupe Type, 1997).

Une fois la décision prise de « sauter dans le train » de l'agrotourisme, il faut orienter son développement sur une base locale et régionale pour créer un produit qui soit typique de son milieu. Pour atteindre ce but, il faut d'abord donner à l'agriculteur la juste place qui lui revient dans l'aventure. La création d'un organisme pour concerter et coordonner le milieu en matière d'agrotourisme semble également nécessaire dans la poursuite d'une démarche tactique et harmonieuse.

\section{Conclusion}

L'agrotourisme représente une avenue de développement intéressante pour plusieurs régions du Québec présentement aux prises avec des problèmes majeurs de dévitalisation (Vachon, 1993) de leur milieu respectif (exode des jeunes, taux de chômage élevés, fermetures d'institutions, etc.). Évidemment, l'agrotourisme ne réglera pas tous les maux sociaux, mais son développement stratégique pourrait contribuer à redynamiser des communautés pour lesquelles l'apport économique de cette activité serait salutaire, tout en participant à redonner leur fierté à des gens laissés de côté par les courants de la nouvelle économie.

La principale épreuve à relever dans le développement de l'agrotourisme sur le territoire réside sans doute dans la particularisation des différentes régions du Québec. Il faut à tout prix éviter le copiage d'une recette gagnante à la grandeur de la province, pour favoriser la différenciation par des traits particuliers et distinctifs propres à chaque région. N'oublions pas que c'est la spécificité et le caractère unique des localités qui font, à coup sûr, leur charme irrésistible.

Martin Cardinal, agent de développement rural au Centre local de développement Côte-de-Beaupré, est bachelier en administration des affaires de l'Université du Québec à Chicoutimi et détient un certificat en gestion du développement touristique de l'Université Laval. Il poursuit actuellement des études supérieures en gestion et planification du tourisme à l'Université du Québec à Montréal.

\section{Bibliographie}

Association des centres locaux de développement du Québec (2001), www.acldq.qc.ca.

Demers, Jacques (1987), Le développement touristique: notions et principes, Québec, $342 \mathrm{p}$.

Duhaime, C., G. Kindra, M. Laroche, et T.E. Muller (1996), Le comportement du consommateur ( $2^{\mathrm{e}}$ édition), Boucherville, Gaëtan Morin éditeur, p. 179-185.

Fédération des agricotours du Québec (1984), Plan d'orientation générale, p. 57-58.

Girardville, Jean-Marie, Lucie Dumas, et Clément Lalancette (1997), Le Québec à votre table. Tremplin Québec: Réseau agrotourisme, Recueil des conférences, Coaticook, 13 et 14 novembre.

Le Groupe Type (1997), Étude sur le tourisme rural relié au monde agricole, Recherche commanditée par l'Union des producteurs agricoles du Québec, le ministère de l'Agriculture, des Pêcheries et de l'Alimentation et Tourisme Québec.

Ministère des Régions (2001), www.mreg.gouv.qc.ca.

Vachon, Bernard (1993), Le développement local : théorie et pratique, Boucherville, Gaëtan Morin éditeur, $331 \mathrm{p}$.

Zins, Michel (1997), Le Québec à votre table. Tremplin Québec: Réseau agrotourisme, Recueil des conférences, Coaticook, 13 et 14 novembre. 